Pacific Journal of Mathematics

STRONGLY CONTINUOUS MARKOV PROCESSES 


\title{
STRONGLY CONTINUOUS MARKOV PROCESSES
}

\author{
S. R. FOGUEL
}

Introduction. This paper is a continuation of [3]. We deal here with Markov processes with continuous parameter, while in [3] the discrete parameter case was studied. The notion of a "Markov Process" (here and in [3]) is different from the standard one: A stationary probability measure is assumed to exist, but the Chapman-Kolmogoroff Equation is replaced by a weaker condition. The exact definitions are given in $\S 1$.

All problems are discussed from a Hilbert space point of view and convergence will mean, always, either strong of weak convergence.

1. Notation and background. We shall repeat here, for completeness, the notation of [3] and some of the results.

Let $(\Omega, \Sigma, \mu)$ be a given measure space where $\mu(\Omega)=1$, and $\mu \geqq 0$. The measure will be called the probability measure. The space of real square integrable functions is denoted by $L_{2}$.

Let $X_{t}(\omega)$ be a family of measurable real functions where $0 \leqq t<\infty$ and $\omega \in \Omega$. This will be called the Markov process and we assume:

If $A$ is a Borel set on the real line and $t_{1}<t_{2}<t_{3}$ then the conditional probability that $X_{t_{3}} \in A$ given $X_{t_{1}}$ and $X_{t_{2}}$ is equal to the conditional probability that $X_{t 3} \in A$ given $X_{t_{2}}$.

Also we assume that the process is stationary. Namely:

$$
\mu\left(X_{t_{1}+s} \in A_{1} \cap X_{t_{2}+s} \in A_{2}\right)=\mu\left(X_{t_{1}} \in A_{1} \cap X_{t_{2}} \in A_{2}\right)
$$

for all $t_{1}, t_{2}, s$ positive real numbers and $A_{1} A_{2}$ Borel sets.

For any set $\sigma \subset \Omega, \chi_{\sigma}$ denotes the characteristic function of this set. Let $B_{t}$ be the closed subspace of $L_{2}$ generated by the functions $\chi_{x_{t}} \in_{A}$. The self adjoint projection on $B_{t}$ is denoted by $E_{t}$. Finally, let $T_{t}$ be the transformation from $B_{0}$ to $B_{t}$ defined by

$$
T_{t} \chi_{x_{0} \in A}=\chi_{x_{t} \in A}
$$

where we used additivity to extend it to whole of $B_{0}$. In [3] the following equations are proved:
1.1
$E_{t_{1}} E_{t_{2}} E_{t_{3}}=E_{t_{1}} E_{t_{3}}$
if $t_{1}<t_{2}<t_{3}$.
$1.2 \mathrm{a}$.
$\left\|T_{t} x\right\|=\|x\|$,
for $x \in B_{0}$.

Received August 3, 1960. This paper was supported by a contract from the National Science Foundation. 
b.

$$
\begin{gathered}
T_{t} B_{0}=B_{t} . \\
\left(T_{t_{1}+s} x, T_{t_{2}+s} y\right)=\left(T_{t_{1}} x, T_{t_{2}} y\right), \quad \text { for } x \in B_{0} y \in B_{0} .
\end{gathered}
$$

c.

See Theorem 2.1 and Lemma 2.4.

Let $P_{t}$ be the operator on $B_{0}$ defined by $P_{t}=E_{0} T_{t}$.

TheOREM 1.1. The operators $P_{t}$ form a semi'group of contractions on $B_{0}$. The adjoint semi group is given by $P_{t}^{*}=T_{t}^{-1} E_{t}$.

Proof. It is clear that $\left\|P_{t}\right\| \leqq 1$. Let $x$ and $y$ be vectors of $B_{0}$ and choose $z \in B_{0}$ so that $T_{s} z=E_{s} y$. Thus $z=T_{s}^{-1} E_{s} y$. Then

$$
\begin{aligned}
\left(P_{s} P_{t} x, y\right) & =\left(E_{0} T_{s} E_{0} T_{t} x, y\right)=\left(T_{s} E_{0} T_{t} x, y\right) \\
& =\left(T_{s} E_{0} T_{t} x, E_{s} y\right)=\left(E_{0} T_{t} x, z\right)=\left(T_{t} x, z\right) .
\end{aligned}
$$

Where we used Equation 1.2c. On the other hand

$$
\begin{aligned}
\left(P_{s+t} x, y\right) & =\left(E_{0} T_{s+t} x, y\right)=\left(E_{0} E_{s} T_{s+t} x, y\right)=\left(E_{s} T_{s+t} x, y\right) \\
& =\left(T_{s+t} x, E_{s} y\right)=\left(T_{s+t} x, T_{s} z\right)=\left(T_{t} x, z\right) .
\end{aligned}
$$

Here we used Equations 1.1 and 1.2c. Now

$$
\left(P_{s} x, y\right)=\left(T_{s} x, y\right)=\left(T_{s} x, E_{s} y\right)=(x, z)=\left(x, T_{s}^{-1} E_{s} y\right) .
$$

The fact that $P_{t}$ is a semi group is our version of the ChapmanKolmogoroff Equation.

In most of this paper it will be assumed that the semi group $P_{t}$ is strongly continuous. We shall say, in this case that the Markov process is strongly continuous.

THEOREM 2.1. The Markov process is strongly continuous if and only if

$$
\lim _{t \rightarrow 0} \mu\left(X_{0} \in A \cap X_{t} \in A\right)=\mu\left(X_{0} \in A\right) .
$$

Proof. Note that

$$
\begin{gathered}
\mu\left(X_{0} \in A\right)=\left\|\chi_{x_{0} \in_{A}}\right\|^{2} \\
\mu\left(X_{0} \in A \cap X_{t} \in A\right)=\left(T_{t} \chi_{x_{0} \in_{A}}, \chi_{x_{0} \epsilon_{A}}\right)=\left(P_{t} \chi_{X_{0} \in_{A}}, \chi_{X_{0} \in_{A}}\right) .
\end{gathered}
$$

Thus

$$
\mu\left(X_{0} \in A\right)-\mu\left(X_{0} \in A \cap X_{t} \in A\right)=\left(\chi_{x_{0} \epsilon_{A}}-P_{t} \chi_{x_{0} \epsilon_{A}}, \chi_{x_{0} \epsilon_{A}}\right)
$$

and this converges to zero if $P_{t}$ converges to the identity operator strongly. On the other hand 


$$
\begin{aligned}
& \left\|P_{t} \chi_{x_{0} \in_{A}}-\chi_{x_{0} \in A}\right\|^{2}=\left\|P_{t} \chi_{X_{0} \in_{A}}\right\|^{2}+\left\|\chi_{x_{0} \in_{A}}\right\|^{2}-2\left(P_{t} \chi_{X_{0} \in_{A}}, \chi_{X_{0} \in_{A}}\right) \\
& \leqq 2\left(\left\|\chi_{x_{0} \in A}\right\|^{2}-\left(P_{t} \chi_{x_{0} \in A}, \chi_{x_{0} \in A}\right)\right) \\
& =2\left(\mu\left(X_{0} \in A\right)-\mu\left(X_{0} \in A \cap X_{t} \in A\right)\right) \text {. }
\end{aligned}
$$

Thus the condition of the Theorem implies that $P_{t} x$ converges to $x$ for a set of functions, $x$, that $\operatorname{span} B_{0}$ and because $\left\|P_{t}\right\| \leqq 1$ this must hold for every $x$ in $B_{0}$.

2. Limit of transition probabilities as $t \rightarrow \infty$. This section is an extension of $\S 3$ of [3]. Throughout this section we assume:

Condition D. There exist a finite a measure $\rho$, on the real line, and an $\varepsilon>0$ such that if $A$ is a Borel set and $\mathcal{P}(A)<\varepsilon$ then

$$
E_{0} \chi_{x t \in A} \neq \chi_{x t \in A} .
$$

This condition was given in [3] and is similar to Doeblin's condition as given in [1] page 192. Another form of the condition is: if $\varphi(A)<\varepsilon$ then

$$
\left\|T_{t} \chi_{x_{0} \epsilon_{A}}\right\|^{2}=\left\|\chi_{x_{0} \in_{A}}\right\|^{2}>\left\|P_{t} \chi_{x_{0} \epsilon_{A}}\right\|^{2} .
$$

In this form it is seen immediately that $t$ can be replaced by any larger number. Thus one can choose $t$ to be of the form $n \delta$ for any fixed $\delta>0$. ( $n$ a positive integer). For a fixed $\delta>0 X_{n \delta}$ form a discreet Markov process for which a Doeblin condition holds. Let $H_{\delta}$ be the space of all functions in $B_{0}$ such that

$$
x \in \bigcap_{n=0}^{\infty} B_{n \delta}, T_{k \delta} x \in \bigcap_{n=0}^{\infty} B_{n \delta} \quad k=1,2, \cdots .
$$

In [3] Theorem 3.7 it was proved that if $x$ is orthogonal to $H_{\delta}$ then $T_{k \delta} x$ tends weakly to zero as $k$ tends to infinity ( $k$ integer).

Theorem 1.2. $x \in H_{\delta}$ if and only if $T_{t} x=x$ for some $t>0$. Thus $H_{\delta}$ is the same for all $\delta$ and will be denoted by $H$. The space $H$ is generated by a finite number of disjoint characteristic functions and is invariant under $T_{t}$ for all $t>0$.

Proof. It is enough to prove first statement for the rest follows from Theorem 3.8 and Corollary 2 of Theorem 3.11 of [3].

In Corollary 2 of Theorem 3.11 of [3] it was shown that if $x \in H_{\delta}$ then $T_{k \delta} x=x$ for some $x$. Thus it is enough to show that if $T_{t} x=x$ for some $t>0$, then $x \in H_{\delta}$. Now if $T_{t} x=x$ then

$$
\left(T_{t+a} x, T_{a} x\right)=\left(T_{t} x, x\right)=\|x\|^{2}=\left\|T_{a} x\right\|^{2}
$$


Thus

$$
T_{t+a} x=T_{a} x
$$

In particlar

$$
x=T_{\imath} x=T_{2 t} x=\cdots .
$$

Thus

$$
x \in \bigcap_{k=0}^{\infty} B_{t k} .
$$

But by Theorem 2.2 of [3]

$$
\bigcap_{k=0}^{\infty} B_{t k}=\bigcap_{n=0}^{\infty} B_{\delta n}
$$

Now

$$
T_{m \delta} x=T_{m \delta+t} x=T_{m \delta+2 t} x=\cdots
$$

or

$$
T_{m \delta} x \in \bigcap_{k=0}^{\infty} B_{m \delta+k t}=\bigcap_{n=m}^{\infty} B_{n \delta} .
$$

Again by Theorem 2.2 of [3]. Thus it suffices to show that $T_{m \delta} x \in B_{0}$ for then $T_{m \delta} x \in \bigcap_{n=0}^{\infty} B_{n \delta}$ by the same Theorem. Now

$$
\begin{aligned}
\sup _{z \in B_{0},\|z\|=1}\left(T_{m \delta} x, z\right) & =\sup _{\tau^{1} \in B_{k t},\left\|z^{1}\right\|=1}\left(T_{m \delta+k t} x, z^{1}\right) \\
& =\sup _{z^{1} \in B_{k t},\left\|z^{1}\right\|=1}\left(T_{m \delta} x, z^{1}\right)=\left\|T_{m \delta} x\right\|
\end{aligned}
$$

for

$$
T_{m \delta} x \in \bigcap_{n=m}^{\infty} B_{n \delta} \subset B_{k t} \quad \text { if } \quad k t>m \delta .
$$

Thus

$$
T_{m \delta} x \in B_{0} \text { and } x \in H_{\delta} \text {. }
$$

Notice that on $H P_{t}=T_{t}$, and $P_{t}$ is a unitary operator.

In the rest of the paper we shall assume that the process $\left\{X_{t}\right\}$, is strongly continuous.

Lemma 2.2. On the space $H T_{t}$ is the identity operator for all $t$.

Proof. Let $\chi$ be one of the atoms generating $H$. Thus $\chi$ is a characteristic function that is not the sum of two characteristic functions 
in $H$. Let $t$ be so small that $\left(T_{t} \chi, \chi\right) \neq 0$. Now $T_{t} \chi$ is also a characteristic function in $H$ and $\left\|T_{t} \chi\right\|=\|\chi\|$. Thus $T_{t} \chi=\chi$ because $\chi$ is an atom. Also for every $n T_{n t} \chi=P_{n t} \chi=\left(P_{t}\right)^{n} \chi=\chi$, hence $T_{t} \chi=P_{t} \chi=\chi$ for all $t$.

Theorem 3.2. Let $x \in B_{0}$ and let $y$ be the projection of $x$ on $H$, then

$$
\text { weak } \operatorname{limit}_{t \rightarrow \infty} P_{t} x=\text { weak } \operatorname{limit}_{t \rightarrow \infty} T_{t} x=y \text {. }
$$

Proof. By the previous lemma it suffices to show that if $x$ is orthogonal to $H$ then $T_{t} x$ tends weakly to zero. Let $z \in B_{0},\|z\|=1$ be a given vector and let $\varepsilon>0$. Choose $\delta_{0}$ so that $\left\|T_{\delta} x-x\right\| \leqq \varepsilon / 2$ if $\delta \leqq \delta_{0}$. By Theorem 3.7 of [3] if $n$ is large enough then

$$
\left|\left(T_{n \delta_{0}} x, z\right)\right| \leqq \varepsilon / 2 \text {. }
$$

Thus

$$
\begin{aligned}
\left|\left(T_{t} x, z\right)\right| & =\left|\left(\left(T_{t}-T_{n \delta_{0}}\right) x, z\right)+\left(T_{n \delta_{0}} x, z\right)\right| \\
& \leqq \varepsilon / 2+\left\|\left(T_{t}-T_{n \delta_{0}}\right) x\right\| .
\end{aligned}
$$

Now

$$
\begin{aligned}
\left\|\left(T_{t}-T_{n \delta_{0}}\right) x\right\|^{2} & =2\|x\|^{2}-2\left(T_{t} x, T_{n \delta_{0}} x\right) \\
& =2\|x\|^{2}-2\left(T_{t-n \delta_{0}} x, x\right)=\left\|T_{t-n \delta_{0}} x-x\right\|^{2}
\end{aligned}
$$

by Equation 1.2.c. If $n$ is so chosen that

$$
t-n \delta_{0}<\delta_{0} \text { then }\left\|\left(T_{t}-T_{n \delta_{0}}\right) x\right\| \leqq \varepsilon / 2 .
$$

3. Differentiability. In this section we do not assume Condition D. The process $\left\{X_{t}\right\}$ is assumed to be strongly continuous. It is known that in this case the function $P_{t} x$ is differentiable at the origin for $x$ in a dense subset of $B_{0}$. The derivative, $Q$, of $P_{t}$ is an unbounded closed operator. Let $D(Q)$ be the domain of $Q$. The simplest case is when $Q$ is bounded. A necessary and sufficient condition for this is that the semi group $P_{t}$ is continuous in the uniform topology. (See 2 Theorem VIII. 2)

THeOREM 1.3. The operator $Q$ is everywhere defined if and only if the expression

$$
1-\frac{\mu\left(X_{0} \in A \cap X_{t} \in A\right)}{\mu\left(X_{0} \in A\right)}
$$

tends to zero uniformly, for all Borel sets $A$. 
Proof. If $\left\|I-P_{t}\right\| \rightarrow 0$ then

$$
1-\frac{\mu\left(X_{0} \in A \cap X_{t} \in A\right)}{\mu\left(X_{0} \in A\right)}=\frac{\left(\chi_{x_{0} \in A}-P_{t} \chi_{x_{0} \in A}, \chi_{x_{0} \in \epsilon_{A}}\right)}{\left\|\chi_{x_{0} \in A}\right\|^{2}} \leqq\left\|I-P_{t}\right\| .
$$

Thus the condition is necessary. Conversely let

$$
x=\sum a_{\imath} \chi_{i} \text { where } \sum a_{i}^{2}\left\|\chi_{\imath}\right\|^{2}=1 \text { and } \chi_{i}=\chi_{x_{0} \in A_{i}}, A_{i} \cap A_{j}=\phi .
$$

Then

$$
\begin{aligned}
& 1-\left(P_{t} x, x\right)=\sum_{i j} a_{\imath} a_{j}\left(\left(\chi_{i}, \chi_{j}\right)-\left(P_{t} \chi_{i}, \chi_{j}\right)\right) \\
& \quad \leqq\left(\sum_{i, j} a_{i}^{2}\left|\left(\chi_{i}, \chi_{j}\right)-\left(P_{t} \chi_{i}, \chi_{j}\right)\right|\right)^{1 / 2}\left(\sum_{i j} a_{j}^{2}\left|\left(\chi_{i}, \chi_{j}\right)-\left(P_{t} \chi_{i}, \chi_{j}\right)\right|\right)^{1 / 2} .
\end{aligned}
$$

By Schwarz's inequality. Let us consider each term separately.

$$
\sum_{i, j} a_{i}^{2}\left|\left(\chi_{i}, \chi_{j}\right)-\left(P_{t} \chi_{i}, \chi_{j}\right)\right|=\sum_{i} a_{i}^{2} \sum_{j}\left|\left(\chi_{i}, \chi_{j}\right)-\left(P_{t} \chi_{i}, \chi_{j}\right)\right| .
$$

For a fixed $i$ we have

$$
\begin{gathered}
\sum_{j}\left|\left(\chi_{i}, \chi_{j}\right)-\left(P_{t} \chi_{i}, \chi_{j}\right)\right|=\sum_{j \neq i}\left(P_{t} \chi_{i}, \chi_{j}\right)+\left\|\chi_{i}\right\|^{2}-\left(P_{t} \chi_{i}, \chi_{i}\right) \\
=\sum_{j}\left(P_{t} \chi_{i}, \chi_{j}\right)-\left(P_{t} \chi_{i}, \chi_{i}\right)+\left\|\chi_{i}\right\|^{2}-\left(P_{t} \chi_{i}, \chi_{i}\right) \\
=\left(P_{t} \chi_{\imath}, 1\right)-\left(P_{t} \chi_{i}, \chi_{i}\right)+\left\|\chi_{i}\right\|^{2}-\left(P_{t} \chi_{i}, \chi_{i}\right)
\end{gathered}
$$

where 1 is the identity function. Now

$$
\left(P_{t} \chi_{i}, 1\right)=\left(T_{t} \chi_{i}, 1\right)=\left(T_{t} \chi_{i}, T_{t} 1\right)=\left(\chi_{i}, 1\right)=\left\|\chi_{i}\right\|^{2} .
$$

Thus the sum over $j$ is equal to

$$
2\left\|\chi_{i}\right\|^{2}\left(1-\frac{\left(P_{t} \chi_{i}, \chi_{i}\right)}{\left\|\chi_{i}\right\|^{2}}\right)
$$

and

$$
\begin{gathered}
\sum_{i, j} a_{i}^{2}\left|\left(\chi_{i}, \chi_{j}\right)-\left(P_{t} \chi_{i}, \chi_{j}\right)\right| \leqq 2 \sup _{i}\left(1-\frac{\left(P_{t} \chi_{i}, \chi_{i}\right)}{\left\|\chi_{i}\right\|^{2}}\right) \\
\sum a_{i}^{2}\left\|\chi_{i}\right\|^{2}=2 \sup \left(1-\frac{\left(P_{t} \chi_{i}, \chi_{i}\right)}{\left\|\chi_{i}\right\|^{2}}\right)
\end{gathered}
$$

For the second term we get

$$
\sum a_{j}^{2}\left|\left(\chi_{i}, \chi_{j}\right)-\left(P_{t} \chi_{i}, \chi_{j}\right)\right|=\sum_{j} a_{j}^{2} \sum_{i}\left|\left(\chi_{i}, \chi_{j}\right)-\left(P_{t} \chi_{i}, \chi_{j}\right)\right|
$$

and 


$$
\begin{aligned}
\sum_{i} \mid & \left(\chi_{i}, \chi_{j}\right)-\left(P_{t} \chi_{i}, \chi_{j}\right) \mid=\left\|\chi_{j}\right\|^{2}-\left(P_{t} \chi_{j}, \chi_{j}\right)+\sum_{i \neq j}\left(P_{t} \chi_{i}, \chi_{j}\right) \\
& =\left\|\chi_{j}\right\|^{2}-\left(P_{t} \chi_{j}, \chi_{j}\right)+\sum_{i}\left(P_{t} \chi_{i}, \chi_{j}\right)-\left(P_{t} \chi_{j}, \chi_{j}\right) \\
& =\left\|\chi_{j}\right\|^{2}-\left(P_{t} \chi_{j}, \chi_{j}\right)+\left(P_{t} 1, \chi_{j}\right)-\left(P_{t} \chi_{j}, \chi_{j}\right) \\
& =2\left(\left\|\chi_{j}\right\|^{2}-\left(P_{t} \chi_{j}, \chi_{j}\right)\right) .
\end{aligned}
$$

And the second term has the same bound. Thus

$$
1-\left(P_{t} x, x\right) \leqq 2 \sup \left(1-\frac{\left(P_{t} \chi_{i}, \chi_{i}\right)}{\left\|\chi_{i}\right\|^{2}}\right) .
$$

Now

$$
\begin{aligned}
\left\|P_{t} x-x\right\|^{2} & =\left\|P_{t} x\right\|^{2}+\|x\|^{2}-2\left(P_{t} x, x\right) \\
& \leqq 2\left(\left(I-P_{t}\right) x, x\right) \leqq 4 \sup _{i}\left(1-\frac{\left(P_{t} \chi_{i}, \chi_{i}\right)}{\left\|\chi_{i}\right\|^{2}}\right) .
\end{aligned}
$$

By assumption this tends to zero uniformly. Hence $\left\|P_{t} x-x\right\|$ tends to zero uniformly, for $x$ in a dense subset of $B_{0}$, and hence everywhere because $\left\|P_{t}\right\| \leqq 1$.

REMARKS. It is enough to assume the condition of the Theorem for a family of Borel sets, $A$, such that the functions $\chi_{A}$ generate $B_{0}$. It follows, from the fact that $Q$ is bounded, that

$$
1-\frac{\mu\left(X_{0} \in A \cap X_{t} \in A\right)}{\mu\left(X_{0} \in A\right)} \leqq \text { (const) } t .
$$

Theorem 1.3 is well known for processes with countable state space. A brief discussion of this case is given in [1] page 265.

The function $P_{t} x$ is differentiable for many $x$ 's exen if $Q$ is unbounded. In order to study this we will need:

LEMma 2.3. Let $R_{t}$ be strongly continuous semi group of operators, defined on a reflexive space $X$. If $x \in X$ then $R_{t} x$ is differentiable if the expression $(1 / t)\left\|R_{t} x-x\right\|$ is bounded for all $t$.

This is included in Theorem 10.7.2 of [4]

Let $y \in L_{2}$ and $\Omega_{1}$ be a subset of $\Omega$ such that $\chi_{r_{1} \in B_{0}}$. Then

$$
\left\|E_{0} y\right\|^{2}=\left\|\chi_{\Omega_{1}} \cdot E_{0} y\right\|^{2}+\left\|\chi_{\Omega_{2}} \cdot E_{0} y\right\|^{2}
$$

where $\Omega_{2}=\Omega-\Omega_{1}$. Now $\chi_{\Omega_{1}} \cdot E_{0} y$ is the projection of $y$ on the subspace generated by characteristic function, in $B_{0}$, of subsets of $\Omega_{1}$. Thus

$$
\begin{aligned}
\left\|\chi_{\Omega_{1}} \cdot E_{0} y\right\|= & \sup \left\{\sum\left(y, \chi_{i}\right) a_{i} \mid \chi_{i}=\chi_{x_{0} \in A_{i}} \in B_{0} \text { and } A_{i}\right. \text { are disjoint } \\
& \text { Borel sets, such that } \left.X_{0} \in A_{i} \subset \Omega_{1} \text {, and } \sum a_{i}^{2}\left\|\chi_{i}\right\|^{2}=1\right\} .
\end{aligned}
$$


But

$$
\left|\sum\left(y, \chi_{i}\right) a_{i}\right| \leqq \sum \frac{\left|\left(y, \chi_{i}\right)\right|}{\left\|\chi_{i}\right\|}\left|a_{i}\right|\left\|\chi_{i}\right\| \leqq\left(\sum \frac{\left(y, \chi_{i}\right)^{2}}{\left\|\chi_{i}\right\|^{2}}\right)^{1 / 2}
$$

Hence

$$
\left\|\chi_{\Omega_{1}} \cdot E_{0} y\right\|^{2}=\sup \left\{\sum \frac{\left(y, \chi_{i}\right)^{2}}{\left\|\chi_{i}\right\|^{2}} \mid \chi_{i}=\chi_{x_{0} \in A_{i}} \in B_{0},\right.
$$

$A_{i}$ disjoint Borel sets and $\left.X_{0} \in A_{i} \subset \Omega_{1}\right\}$

A similar expresion holds for $\left\|\chi_{\Omega_{2}} \cdot E_{0} y\right\|^{2}$.

Theorem 3.3. Let $A$ be a Borel set. The function $P_{t} \chi_{x_{0} \in \in_{A}}$ is differentiable at zero if and only if the two expressions below, are bounded:

1. $\frac{1}{t^{2}} \sup \left\{\sum \frac{\mu\left(X_{t} \in A \cap X_{0} \in A_{i}\right)^{2}}{\mu\left(X_{0} \in A_{i}\right)} \mid A_{i}\right.$ disjoint

$$
\text { Borel sets and } \left.A_{i} \cap A=\phi\right\} \text {. }
$$

2. $\frac{1}{t^{2}} \sup \left\{\sum \frac{\left(\mu\left(X_{t} \in A \cap X_{0} \in A_{i}\right)-\mu\left(X_{0} \in A_{i}\right)\right)^{2}}{\mu\left(X_{0} \in A_{i}\right)} \mid A_{i}\right.$ disjoint

$$
\text { Borel sets and } \left.A_{i} \subset A\right\} \text {. }
$$

Proof. By Lemma 2.3 and the above discussion it is enough to show that

$$
\frac{1}{t^{2}} \sup \left\{\sum \frac{\left(P_{t} \chi_{x_{0} \in A}-\chi_{x_{0} \in A}, \chi_{x_{0} \epsilon_{A i}}\right)^{2}}{\left\|\chi_{X_{0} \in A i}\right\|^{2}} \mid A_{i} \text { disjoint and } A_{i} \cap A=\phi\right\}
$$

and

$$
\frac{1}{t^{2}} \sup \left\{\sum \frac{\left(P_{t} \chi_{x_{0} \epsilon_{A}}-\chi_{x_{0} \epsilon_{A}}, \chi_{x_{0} \epsilon_{A_{i}}}\right)}{\left\|\chi_{x_{0} \epsilon_{i}}\right\|^{2}} \mid A_{i} \text { disjoint and } A_{i} \subset A\right\}
$$

are both bounded. But these expressions are equal to 1 and 2 respectively.

REMARK. If $A$ is an atom for $B_{0}$ then the second expression is

$$
\begin{aligned}
& \frac{1}{t^{2}}\left(\frac{\chi\left(X_{t} \in A \cap X_{0} \in A\right)-\mu\left(X_{0} \in A\right)}{\mu\left(X_{0} \in A\right)}\right)^{2} \mu\left(X_{0} \in A\right) \\
& =\left(\frac{1}{t}\left(1-\frac{\mu\left(X_{t} \in A \cap X_{0} \in A\right)}{\mu\left(X_{0} \in A\right)}\right)\right)^{2} \mu\left(X_{0} \in A\right) .
\end{aligned}
$$


A more precise information is available in the following special case.

TheOREM 4.3. Let $x \in B_{0}$. Then $x \in D(Q)$ and $(Q x, x)=0$ if and only if $\left(1 / t^{2}\right)\left(\|x\|^{2}-\left(P_{t} x, x\right)\right)$ is bounded. In this case $Q^{*} x$ exists and is equal to $-Q x$.

Proof. If $y \in B_{0}$ then

$$
\begin{aligned}
\left\|y-P_{t} y\right\|^{2} & =\|y\|^{2}+\left\|P_{t} y\right\|^{2}-2\left(P_{t} y, y\right) \\
& \leqq 2\left(\|y\|^{2}-\left(T_{t} y, y\right)\right)=\left\|y-T_{t} y\right\|^{2}
\end{aligned}
$$

thus

a.

$$
\frac{\left\|T_{t} y-y\right\|}{\sqrt{t}}=\sqrt{2 \frac{\left(y-P_{t} y, y\right)}{t}} \geqq \frac{\left\|P_{t} y-y\right\|}{\sqrt{t}} .
$$

Also if $y$ and $z$ are any two vectors in $B_{0}$ then

b.

$$
\begin{aligned}
\left(\frac{1}{t}\left(P_{t}-1\right) z, y\right) & =\frac{1}{t}\left(T_{t} z-z, y\right)=\frac{1}{t}\left(T_{t} z, y-T_{t} y\right) \\
& =\frac{1}{t}\left(T_{t} z-z, y-T_{t} y\right)+\frac{1}{t}\left(z, y-P_{t} y\right)
\end{aligned}
$$

where we used Equation 1.2.c for the third equality.

Let $x$ be such that $\left(1 / t^{2}\right)\left(\|x\|^{2}-\left(P_{t} x, x\right)\right)$ is bounded. Then from (a) we get

$$
\left\|\frac{1}{t^{2}}\left(P_{t} x-x\right)\right\|^{2} \leqq 2 \frac{\left(x-P_{t} x, x\right)}{t^{2}}
$$

and is bounded by assumption. Thus we know from Lemma 2.3 that $x \in D(Q)$. Moreover

$$
(Q x, x)=-\lim t \frac{\left(x-P_{t}, x\right)}{t^{2}}=0 .
$$

Conversely let $x \in D(Q)$ and $(Q x, x)=0$. If $y \in D(Q)$ then it follows from (b) that

$$
\begin{aligned}
(Q x, y) & =\lim _{t \rightarrow 0} \frac{1}{t}\left(\left(P_{t}-1\right) x, y\right) \\
& =\lim _{t \rightarrow 0} \frac{1}{t}\left(T_{t} x-x, y-T_{t} y\right)+\frac{1}{t}\left(x, y-P_{t} y\right)
\end{aligned}
$$

the second term tends to $-(x, Q y)$ while the first is bounded by 


$$
\begin{aligned}
\left|\frac{1}{t}\left(T_{t} x-x, y-T_{t} y\right)\right| & \leqq \frac{\left\|T_{t} x-x\right\|}{\sqrt{t}} \frac{\left\|y-T_{t} y\right\|}{\sqrt{t}} \\
& =\left(2 \frac{\left(x-P_{t} x, x\right)}{t} \cdot 2 \frac{\left(y-P_{t} y, y\right)}{t}\right)^{1 / 2}
\end{aligned}
$$

as $t \rightarrow 0$ this tends to

$$
(4(Q x, x)(Q y, y))^{1 / 2}=0
$$

Thus

$$
(Q x, y)=-(x, Q y)
$$

or

$$
x \in D\left(Q^{*}\right) \text { and } Q^{*} x=-Q x \text {. }
$$

Now

$$
\begin{aligned}
\left(x-P_{t} x, x\right) & =\int_{0}^{t}\left(Q P_{u} x, x\right) d u \leqq t \max _{u \leqq t}\left|\left(Q P_{u} x, x\right)\right| \\
& =t \max _{u \leqq t}\left|\left(P_{u} x, Q x\right)\right|=t \max _{u \leqq t}\left|\left(P_{u} x-x, Q x\right)\right| \\
& \leqq \text { const. } t^{2}
\end{aligned}
$$

because $\left\|P_{u} x-x\right\| \leqq$ const. $u$.

REMARK. If $x$ is a characteristic function then it is easy to see that $Q x=0$ if $(Q x, x)=0$.

The referee called my attention to the fact that this theorem generalizes to arbitrary semi groups of contraction operators, when $T_{t}$ is replaced by the group of unitary operators which project down to $P_{t}$ as in $s_{z}$ Nagy theorem (See Riesz Nagy appendix to the third edition). Some simple changes have to be done to take care of the complex case.

\section{BIBLIOGRAPHY}

1. J. L. Doob, Stochastic Processes, Wiley, New York, 1953.

2. J. T. Schwartz and N. Dunford, Linear Operators. Interscience, New York, 1958.

3. S. R. Foguel, Weak and strong convergence of Markov Processes, Pacific J. Math., 10 (1960), 1221-1234.

4. E. Hille and R. S. Phillips, Functional analysis and semi groups, Amer. Math. Soc. New York, 1957.

UNIVERSITY OF CALIFORNIA

BERKELEY 


\title{
PACIFIC JOURNAL OF MATHEMATICS
}

\section{EDITORS}

\author{
Ralph S. Phillips \\ Stanford University \\ Stanford, California \\ F. H. BrownelL \\ University of Washington \\ Seattle 5 , Washington
}

A. L. Whiteman

University of Southern California

Los Angeles 7. California

L. J. PAIGE

University of California

Los Angeles 24, California

\section{ASSOCIATE EDITORS}

\author{
E. F. BECKENBACH \\ T. M. CHERRY
} \\ D. DERRY \\ M. OHTSUKA \\ UNIVERSITY OF BRITISH COLUMBIA \\ CALIFORNIA INSTITUTE OF TECHNOLOGY \\ UNIVERSITY OF CALIFORNIA \\ MONTANA STATE UNIVERSITY \\ UNIVERSITY OF NEVADA \\ NEW MEXICO STATE UNIVERSITY \\ OREGON STATE COLLEGE \\ UNIVERSITY OF OREGON \\ OSAKA UNIVERSITY \\ UNIVERSITY OF SOUTHERN CALIFORNIA
}

H. L. ROYDEN

E. SPANIER
E. G. STRAUS

F. WOLF

\section{SUPPORTING INSTITUTIONS}

\author{
STANFORD UNIVERSITY \\ UNIVERSITY OF TOKYO \\ UNIVERSITY OF UTAH \\ WASHINGTON STATE COLLEGE \\ UNIVERSITY OF WASHINGTON \\ AMERICAN MATHEMATICAL SOCIETY \\ CALIFORNIA RESEARCH CORPORATION \\ HUGHES AIRCRAFT COMPANY \\ SPACE TECHNOLOGY LABORATORIES \\ NAVAL ORDNANCE TEST STATION
}

Mathematical papers intended for publication in the Pacific Journal of Mathematics should be typewritten (double spaced), and the author should keep a complete copy. Manuscripts may be sent to any one of the four editors. All other communications to the editors should be addressed to the managing editor, L. J. Paige at the University of California, Los Angeles 24, California.

50 reprints per author of each article are furnished free of charge; additional copies may be obtained at cost in multiples of 50 .

The Pacific Journal of Mathematics is published quarterly, in March, June, September, and December. The price per volume (4 numbers) is $\$ 12.00$; single issues, $\$ 3.50$. Back numbers are available. Special price to individual faculty members of supporting institutions and to individual members of the American Mathematical Society: $\$ 4.00$ per volume; single issues, $\$ 1.25$.

Subscriptions, orders for back numbers, and changes of address should be sent to Pacific Journal of Mathematics, 103 Highland Boulevard, Berkeley 8, California.

Printed at Kokusai Bunken Insatsusha (International Academic Printing Co., Ltd.), No. 6 , 2-chome, Fujimi-cho, Chiyoda-ku, Tokyo, Japan.

PUBLISHED BY PACIFIC JOURNAL OF MATHEMATICS, A NON-PROFIT CORPORATION

The Supporting Institutions listed above contribute to the cost of publication of this Journal, but they are not owners or publishers and have no responsibility for its content or policies. 


\section{Pacific Journal of Mathematics}

\section{Vol. 11, No. $3 \quad$ BadMonth, 1961}

Errett Albert Bishop, A generalization of the Stone-Weierstrass theorem ..........

Hugh D. Brunk, Best fit to a random variable by a random variable measurable with

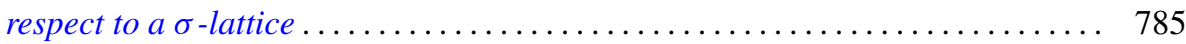

D. S. Carter, Existence of a class of steady plane gravity flows . . . . . . . . . . 803

Frank Sydney Cater, On the theory of spatial invariants ............... 821

S. Chowla, Marguerite Elizabeth Dunton and Donald John Lewis, Linear



Paul Civin and Bertram Yood, The second conjugate space of a Banach algebra as



William J. Coles, Wirtinger-type integral inequalities .

Shaul Foguel, Strongly continuous Markov processes ....................

David James Foulis, Conditions for the modularity of an orthomodular lattice ...... Jerzy Górski, The Sochocki-Plemelj formula for the functions of two complex variables.

John Walker Gray, Extensions of sheaves of associative algebras by non-trivial

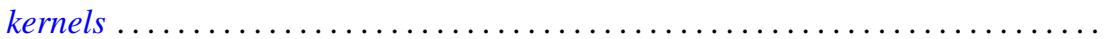

Maurice Hanan, Oscillation criteria for third-order linear differential equations .... 919 Haim Hanani and Marian Reichaw-Reichbach, Some characterizations of a class of unavoidable compact sets in the game of Banach and Mazur .............

John Grover Harvey, III, Complete holomorphs . . . . . . . . . . . . . . . . 961 Joseph Hersch, Physical interpretation and strengthing of M. Protter's method for vibrating nonhomogeneous membranes; its analogue for Schrödinger's

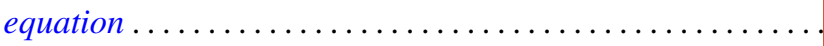

James Grady Horne, Jr., Real commutative semigroups on the plane...

Nai-Chao Hsu, The group of automorphisms of the holomorph of a group...

F. Burton Jones, The cyclic connectivity of plane continua

John Arnold Kalman, Continuity and convexity of projections and barycentric

coordinates in convex polyhedra

Samuel Karlin, Frank Proschan and Richard Eugene Barlow, Moment inequalities of

Pólya frequency functions .

Tilla Weinstein, Imbedding compact Riemann surfaces in 3-space. .

Azriel Lévy and Robert Lawson Vaught, Principles of partial reflection in the set

theories of Zermelo and Ackermann

Donald John Lewis, Two classes of Diophantine equations .....

Daniel C. Lewis, Reversible transformations . . .

Gerald Otis Losey and Hans Schneider, Group membership in rings and

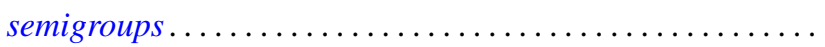

M. N. Mikhail and M. Nassif, On the difference and sum of basic sets of

polynomials.

Alex I. Rosenberg and Daniel Zelinsky, Automorphisms of separable algebras .

Robert Steinberg, Automorphisms of classical Lie algebras .... 